\title{
Nebulised colistin for ventilator-associated pneumonia prevention
}

\author{
Marios Karvouniaris ${ }^{1}$, Demosthenes Makris ${ }^{1}$, Paris Zygoulis ${ }^{1}$, \\ Apostolos Triantaris ${ }^{1}$, Stelios Xitsas ${ }^{2}$, Konstantinos Mantzarlis ${ }^{1}$, \\ Efthimia Petinaki ${ }^{2}$ and Epaminondas Zakynthinos ${ }^{1}$
}

Affiliations: ${ }^{1}$ Critical Care Dept, University of Thessaly, University Hospital of Larissa, Larissa, Greece. ${ }^{2}$ Microbiology Laboratory, University of Thessaly, University Hospital of Larissa, Larissa, Greece.

Correspondence: Demosthenes Makris, Biopolis, 41110 Larissa, Greece. E-mail: appollon7ahotmail.com

ABSTRACT We evaluated whether prophylactic nebulised colistin could reduce ventilator-associated pneumonia (VAP) rates in an intensive care unit (ICU) setting with prevalent multidrug-resistant (MDR) bacteria.

We used a single-centre, two-arm, randomised, open-label, controlled trial in a 12-bed ICU in the University Hospital of Larissa, Greece. Patient inclusion criteria included mechanical ventilation of $>48 \mathrm{~h}$. The two arms consisted of prophylaxis with $500000 \mathrm{U}$ colistin (Col group) or normal saline (NS group), thrice daily, for the first 10 ICU days or until extubation. The primary outcome of the study was the 30-day VAP incidence.

In total, 168 patients entered the study. VAP incidence was not different between Col and NS group patients (14 (16.7\%) versus $25(29.8 \%)$, respectively, $\mathrm{p}=0.07)$. Regarding the secondary outcomes, the intervention resulted in a lower VAP incidence density rate (11.4 versus 25.6 , respectively, $\mathrm{p}<0.01$ ), and less Gram-negative bacteria-VAP ( $\mathrm{p}=0.03)$ and MDR-VAP $(\mathrm{p}=0.04)$. Among VAP patients $(\mathrm{n}=39)$, prophylaxis with inhaled colistin improved ICU survival $(\mathrm{p}=0.016)$. There was no evidence of increased resistance to colistin or multidrug resistance.

Our findings suggest that nebulised colistin had no significant effect on VAP incidence.

@ERSpublications

In a RCT, nebulised colistin prophylaxis did not decrease ventilator-associated pneumonia incidence http://ow.ly/R8FOE

For editorial comments see Eur Respir J 2015; 46: 1544-1547 [DOI: 10.1183/13993003.01361-2015].

This article has supplementary material available from erj.ersjournals.com

Received: March 122014 | Accepted after revision: July 072015 | First published online: Sept 242015

Clinical trial: This study is registered at ClinicalTrials.gov with identifier number NTC01025921.

Support statement: The study was supported by the University of Thessaly (UT843).

Conflict of interest: None declared.

Copyright OERS 2015 


\section{Introduction}

Nosocomial infections such as ventilator-associated pneumonia (VAP) cause serious morbidity in critical care patients [1-3]. Patients with burns, severe trauma and neurosurgical causes of admission are at increased risk to present respiratory infections $[1,4-6]$, whereas recent exposure either to antibiotics or the healthcare environment $[4,7]$ is a risk factor for multidrug-resistant (MDR) respiratory infections.

Prophylactic topical application of antibiotics on the tracheobronchial tree might be a preventive strategy for reducing VAP in intensive care unit (ICU) patients [8]. Previous studies have evaluated the use of polymyxin instilled to the trachea or sprayed in the pharynx for preventing Gram-negative bacteria (GNB)-pneumonia [9-11]. The intervention was associated with reduced pneumonia rates, although it did not lead to increasing rates of polymyxin-resistant microorganisms [9, 10]. However, other authors reported elevated numbers of pneumonia with colistin (polymyxin E)-resistant bacteria and concluded that this strategy might be "dangerous" [11]. Hence, there is scepticism about using such antibiotics, topically applied in the bronchial tree, to prevent VAP. Furthermore, available data on this issue come mainly from studies in critical care settings with low levels of bacterial resistance to antibiotics [9-11].

In the present randomised controlled study, we aimed to investigate the impact of the prophylactic use of colistin, applied by nebulisation, on the incidence of VAP. We studied a population in a critical care setting where MDR-GNB were predominant and endemic $[6,12,13]$.

\section{Materials and methods}

Design and population

This was a single-centre, two-arm, open-label randomised controlled study conducted in a 12-bed ICU between November 2011 and July 2013. All consecutive patients entered the study if they fulfilled the following criteria: age $>18$ years and mechanical ventilation $>48 \mathrm{~h}$. Exclusion criteria were: grossly purulent sputum or pneumonia on admission, new and persistent infiltrates on chest radiography within $48 \mathrm{~h}$ from admission, severe chronic obstructive pulmonary disease (COPD) [14], pregnancy, allergy to colistin, and colonisation or infection with a strain resistant to colistin on admission.

Patients received either colistin (colistimethate sodium, $500000 \mathrm{U}$, diluted in $2.5 \mathrm{~mL}$ water for injection) (Col group) or $2.5 \mathrm{~mL} 0.9 \%$ saline (NS group), thrice daily. Prophylaxis lasted for the first 10 days of mechanical ventilation or until extubation (whichever occurred first). Nebulisation was performed with the use of a conventional jet nebuliser placed in the inspiratory limb of ventilator tubing behind the Y-piece [15] and continued until the nebuliser deposit became dry.

The occurrence of respiratory infection was not a reason for stopping either colistin or saline. Randomisation was performed with the method of random permuted blocks to achieve balance among the two arms. A block size of four patients was used. Within each block, equal numbers of patients were allocated randomly in each of the two treatment arms. The order of treatments within each block was permuted with the use of random numbers [16]. The ethics committee of the hospital approved the study. Informed consent was obtained, either from the patient or the next of kin. The study was supported by the University of Thessaly (UT843) and registered at http://www.clinicaltrials.gov (NTC01025921).

\section{Outcomes}

We primarily assessed the 30-day VAP incidence. In addition, we assessed ventilator-associated tracheobronchitis (VAT) and airway colonisation incidence, ICU length of stay, ICU mortality, hospital mortality, ventilator-free days, days without systemic antibiotic exposure in the ICU and incidence of colistin-resistant bacteria in tracheobronchial aspirate (TBA) or blood.

\section{Definitions}

VAP and VAT were described as previously reported [6] (for details, see online supplementary material). Only microbiologically confirmed infections, either VAP or VAT, were accounted [17]. Airway colonisation was diagnosed as previously defined [18]. The incidence of respiratory infections was expressed as 1) the ratio (percentage) of patients with VAP to the total number of patients in each arm of the study and 2) the incidence density rate (IDR), which is the total number of respiratory infection cases per 1000 ventilation days [19]. Quantitative and qualitative assessment of TBA was routinely used according to a grading scale based on a previous report [20]. For the definitions of MDR bacteria, mechanical ventilation-free days, appropriateness/exposure to antibiotics and infection control policy, see the online supplementary material.

\section{Safety issues}

The point prevalence of antimicrobial resistance to antibiotics was examined in blood and TBA samples obtained over equal time periods between November 2011 and July 2013 (before, at regular 6-month intervals during the study and 1 month after the assessment of the last recruited patient). 


\section{Statistical analysis}

On the basis of the incidence of VAP in our ICU before the start of the trial (34.6\%) [13], a total sample of 168 patients was required to document a $40 \%$ reduction in VAP occurrence rate ( $\mathrm{p}=0.05$, power $80 \%$ ). An independent data and safety monitoring board periodically reviewed efficacy and safety data.

The study used an intention-to-treat analysis. Comparisons between variables were examined by the Chi-squared test or Fisher's exact test, or by the t-test or Mann-Whitney U-test when appropriate; the analysis of variance method or the Kruskal-Wallis test was used for more than two-group comparisons. For Kaplan-Meier analysis and Cox regression analysis, see the online supplementary material. Only the first VAP episode was included in the analysis; patients who presented both VAT and VAP were considered as VAP cases. All statistical tests were two-sided at a level of significance of $\mathrm{p}<0.05$. Analyses were performed with SPSS for Windows version 15 (IBM, Somers, NY, USA).

\section{Results}

In total, 168 patients entered the study (figure 1). Baseline characteristics of participants before randomisation are presented in table 1 . One half of the patients $(n=84)$ received colistin (Col group) for (median (interquartile range)) $10(5.2-10)$ days and the other half of the patients $(n=84)$ received saline (NS group) for $9(4-10)$ days $(\mathrm{p}=0.25)$. Prophylaxis commenced $6.5(4-9.75)$ and $7(4-10) \mathrm{h}$ following intubation, respectively $(\mathrm{p}=0.50)$. In total, 124 patients were on systemic antibiotics covering GNB during the 10-day period of prophylaxis [64 (76.2\%) versus $60(71.4 \%), \mathrm{p}=0.60]$, respectively; there was no difference between groups with regard to number/types of antibiotics received or their antibiotic treatment duration $(\mathrm{p}>0.5)$.

There were 60 positive TBA isolates in the Col group and 70 positive TBA isolates in the NS group. The first positive TBA culture was presented on ICU day 10 (5-17) and 4 (2.5-8) in the Col and NS groups, respectively (online supplementary figure $\mathrm{S} 1, \mathrm{p}<0.01$ ). There was a predominance of GNB in both arms of the study (online supplementary table S1).

\section{VAP incidence}

Overall, VAP incidence was 23.2\% (table 2); VAP was present in 14 (16.7\%) patients in the Col group compared with $25(29.8 \%)$ patients in the NS group ( $\mathrm{p}=0.07)$.

IDR was 11.4 and 25.6 in the Col and NS groups, respectively $(\mathrm{p}<0.01)$. GNB-VAP rates and MDR-VAP rates, including Acinetobacter baumannii cases, were decreased in the Col group compared with the NS group (table 2).

The VAP rate in patients who received systemic antibiotics covering GNB during the period of prophylaxis was $9 / 64(14.1 \%)$ in the Col group and $19 / 60(31.7 \%)$ in the NS group $(p=0.03)$. The GNB-VAP rate in

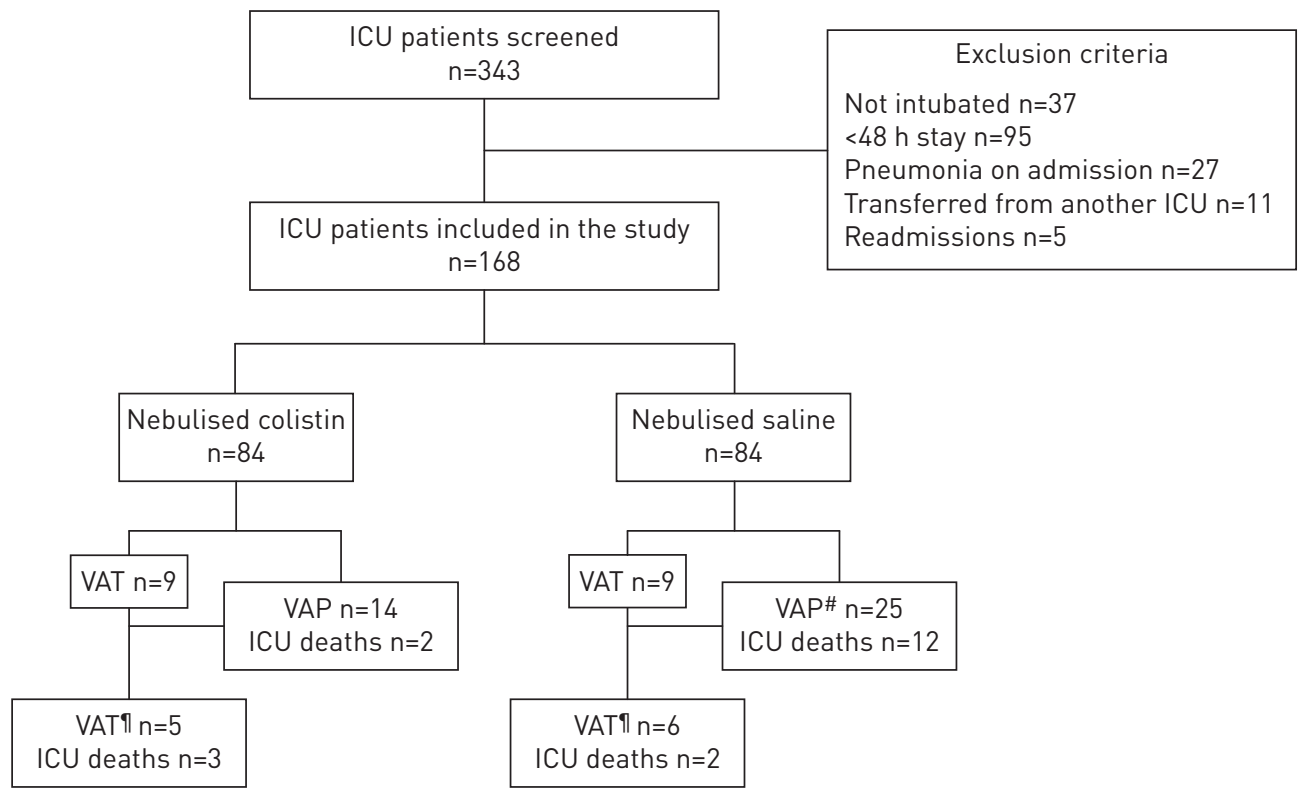

FIGURE 1 Flowchart of the study. ICU: intensive care unit; VAT: ventilator-associated tracheobronchitis; VAP: ventilator-associated pneumonia." : 27 VAP events and one independent VAT event in these 25 patients; " as a final diagnosis. 


\begin{tabular}{|c|c|c|c|c|}
\hline & Overall $n=168$ & Col group $n=84$ & NS group $n=84$ & p-value \\
\hline Age years & $59.5(43-69)$ & $60(44.2-70.5)$ & $58(38.25-67.7)$ & 0.29 \\
\hline Female & $42(25)$ & 20 (23.8) & $22(26.2)$ & 0.86 \\
\hline Emergency ward admission & $81(48.2)$ & $39(46.4)$ & $42(50)$ & 0.76 \\
\hline Infection on admission & $36(21.4)$ & 13 (15.5) & $23(27.4)$ & 0.09 \\
\hline Immunosuppression & $17(10.1)$ & $11(16.2)$ & $6(7.1)$ & 0.31 \\
\hline \multicolumn{5}{|l|}{ Comorbidities } \\
\hline Cirrhosis & $6(3.6)$ & $2(2.4)$ & $4(4.8)$ & 0.68 \\
\hline Heart failure & $7(4.2)$ & $4(4.8)$ & $3(3.6)$ & 1.0 \\
\hline Renal failure on dialysis & $3(1.8)$ & $1(1.2)$ & $2(2.4)$ & 1.0 \\
\hline COPD and bronchial asthma & $20(11.9)$ & $10(11.9)$ & $10(11.9)$ & 1.0 \\
\hline ARDS & $10(6)$ & 7 (8.3) & $3(3.6)$ & 0.33 \\
\hline Nonfatal comorbid disease & $105(62.5)$ & 50 (59.5) & 55 (65.5) & 0.52 \\
\hline Charlson Comorbidity Index & $2(1-4)$ & $2(1-4)$ & $2(0.25-4)$ & 0.53 \\
\hline Antibiotics at admission & 109 (64.9) & $56(66.7)$ & $53(63.1)$ & 0.75 \\
\hline Treatment with inhaled $\beta$-agonists & $22(13.09)$ & 12 (14.28) & $10(11.3)$ & 0.8 \\
\hline Positive TBA culture & $10(6)$ & $3(3.6)$ & 7 (8.3) & 0.33 \\
\hline \multicolumn{5}{|l|}{ Category of admission } \\
\hline Medical & $59(35.1)$ & 26 (31) & 33 (39.3) & 0.33 \\
\hline Surgical & $45(26.8)$ & $23(27.4)$ & $22(26.2)$ & 1.0 \\
\hline Neurosurgical & $64(38.1)$ & $35(41.6)$ & 29 (34.5) & 0.43 \\
\hline APACHE II & $19(15-23)$ & $19(14-23)$ & $19(15-23)$ & 0.96 \\
\hline SOFA & $9(7-11)$ & $9(7-11)$ & $9(7-11)$ & 1.0 \\
\hline White blood cells $\times 10^{3} \cdot \mathrm{mm}^{-3}$ & $12.2(8.9-16.7)$ & $12.9(8.4-16.2)$ & $11.9(9.3-17.4)$ & 0.85 \\
\hline CRP $\mathrm{mg} \cdot \mathrm{dL}^{-1}$ & $3.7(0.8-10.9)$ & $3.4(0.7-10.3)$ & $3.7(1-11.5)$ & 0.36 \\
\hline $\mathrm{PaO}_{2} / \mathrm{FiO}_{2}$ & $273(186-365)$ & $278(186.5-386.5)$ & 272 (181.4-349) & 0.34 \\
\hline
\end{tabular}

Data are presented as $\mathrm{n}(\%)$ or median (interquartile range) unless otherwise stated. Col group: colistin group; NS group: normal saline group; COPD: chronic obstructive pulmonary disease; ARDS: acute respiratory distress syndrome; TBA: tracheobronchial aspirate; APACHE: Acute Physiology and Chronic Health Evaluation; SOFA: Sequential Organ Failure Assessment; CRP: C-reactive protein; $\mathrm{PaO}_{2}$ : arterial oxygen tension; $\mathrm{FiO}_{2}$ : inspiratory oxygen fraction.

patients who received systemic antibiotics with a spectrum covering GNB was 6/64 (9.4\%) in the Col group and $15 / 60(25 \%)$ in the NS group ( $\mathrm{p}=0.03)$.

Cox regression analysis showed that the intervention had a significant impact on the IDR of VAP after adjusting for covariables that were found to be significantly associated with VAP in univariate analysis (online supplementary table S2). The intervention was independently associated with reduced rates of GNB- and MDR-VAP; neurosurgical admission was also associated with an increased risk for GNB-VAP (odds ratio (95\% CI) 2.17 (1.00-4.72), p=0.05) (online supplementary tables S3 and S4).

The evolution of clinical and laboratory indices and scores during the ICU stay is shown in online supplementary figure S2. The Sequential Organ Failure Assessment (SOFA) score was higher in the NS group on the day of VAP diagnosis (day 0) $(\mathrm{p}=0.02)$, and white blood cell count was elevated in the NS group compared with the Col group at days 2, 4 and 6 following VAP diagnosis $(\mathrm{p}<0.05)$.

\section{VAT incidence}

There was no difference between the Col and NS groups with regard to VAT incidence, whereas VAT IDR was 4.1 and 6.6, respectively $(\mathrm{p}<0.01)$ (table 2). Six out of $17(35.2 \%)$ patients with VAT presented later VAP (ranging from 1 to 9 days after the VAT episode) with the same pathogen, but no difference was found between the Col and NS groups $(p=0.68)$.

\section{Mortality and morbidity indices}

There was no difference between the two groups in terms of ICU or hospital mortality (table 2). Among VAP patients, ICU mortality was decreased in the Col group compared with the NS group (7.1\% versus $44 \%, \mathrm{p}=0.028$ ), whereas hospital mortality rates were $14.3 \%$ and $44 \%(\mathrm{p}=0.08)$, respectively; prophylaxis with inhaled colistin was an independent factor for ICU survival in VAP patients in multivariate analysis ( $\mathrm{p}=0.016)$ (for details, see the online supplementary material). 
TABLE 2 Outcomes of participants in the two arms of the study ${ }^{\#}$

Overall $n=168$ Col group $n=84 \quad$ NS group $n=84 \quad p$-value

\begin{tabular}{lcccc}
\hline Primary outcome & & & & \\
VAP & $39(23.2)$ & $14(16.7)$ & $25(29.8)$ & 0.07 \\
Secondary outcomes & & & & \\
VAP IDR & 18 & 11.4 & 25.6 & $<0.01$ \\
GNB-VAP & $30(17.9)$ & $9(10.7)$ & $21(25)$ & 0.03 \\
MDR-VAP & $22(13.1)$ & $6(7.1)$ & $16(19)$ & 0.04 \\
VAP due to Acinetobacter baumannii & $13(7.7)$ & $2(2.8)$ & $11(13.1)$ & 0.02 \\
VAP due to Staphylococcus species & $9(5.4)$ & $5(6)$ & $4(4.8)$ & 1.0 \\
VAP during the 10-day prophylaxis & $28(16.7)$ & $9(10.7)$ & $19(22.6)$ & 0.06 \\
VAP post 10-day prophylaxis & $11(6.5)$ & $5(6)$ & $6(7.1)$ & 1.0 \\
VAP following VAT & $6(3.6)$ & $4(4.8)$ & $2(2.4)$ & 0.68 \\
VAT IDR & 5.3 & 4.1 & 6.6 & $<0.01$ \\
VAT & $11(6.5)$ & $5(6)$ & $6(7.1)$ & 1.0 \\
GNB-VAT & $11(6.5)$ & $5(6)$ & $6(7.1)$ & 1.0 \\
MDR-VAT & $9(5.4)$ & $4(4.8)$ & $5(6)$ & 1.0 \\
VAT due to Acinetobacter baumannii & $5(3)$ & $2(2.4)$ & $3(3.6)$ & 1.0 \\
VAT during the 10-day prophylaxis & $7(4.2)$ & $2(2.4)$ & $5(6)$ & 0.44 \\
Airway colonisation & $34(20.2)$ & $16(19)$ & $18(21.4)$ & 0.85 \\
During the 10-day prophylaxis & $22(13.1)$ & $6(7.1)$ & $16(19)$ & $<0.01$ \\
After the 10-day prophylaxis & $12(7.1)$ & $10(11.9)$ & $2(2.4)$ & $<0.01$ \\
Due to GNB & $33(19.6)$ & $15(17.9)$ & $18(21.4)$ & 0.70 \\
Due to MDR & $28(16.7)$ & $13(15.5)$ & $15(17.9)$ & 0.84 \\
ICU mortality & $54(32.1)$ & $25(29.8)$ & $29(34.5)$ & 0.62 \\
ICU stay days & $13.5(7-28)$ & $16.5(7-29.7)$ & $13(6.25-24.7)$ & 0.31 \\
Hospital mortality & $60(35.7)$ & $29(34.5)$ & $31(36.9)$ & 0.87 \\
Hospital stay days & $20(12-30)$ & $23(12-30)$ & $19(12.2-30)$ & 0.38 \\
MV days & $12(5-21)$ & $13.5(5-24.5)$ & $9(5-18.7)$ & 0.26 \\
MV days before first episode of VAl & $7(4-12.2)$ & $10(7-15)$ & $6(4-10)$ & 0.01 \\
MV days before first episode of VAP & $6(4-12)$ & $10(5.5-16.5)$ & $4(3.5-10.5)$ & 0.07 \\
MV-free days & $1(0-3)$ & $1(0-3)$ & $1(0-3)$ & 0.90 \\
Days without systemic antibiotic exposure & $1(0-5)$ & $2.5(0-5)$ & $0.5(0-3)$ & 0.06 \\
Tracheostomy & $76(45.2)$ & $43(51.2)$ & $33(39.3)$ & 0.16 \\
\hline
\end{tabular}

Data are presented as $\mathrm{n}(\%)$ or median (interquartile range), unless otherwise stated. Col group: colistin group; NS group: normal saline group; VAP: ventilator-associated pneumonia; IDR: incidence density rate of all VAP and all VAT per 1000 total ventilator days (before and after the first respiratory infection); GNB, Gram-negative bacteria; MDR: multidrug-resistant; VAT: ventilator-associated tracheobronchitis; ICU: intensive care unit; MV: mechanical ventilation; VAl: ventilator-associated infection (either VAP or VAT). \#: participants who

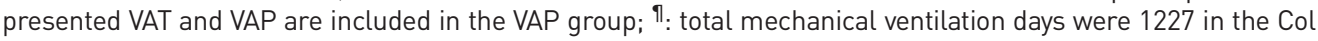
group and 1053 in the NS group.

No difference was found between the two groups with regard to ICU or hospital, or mechanical ventilation duration (table 2). The number of days without systemic antibiotic consumption in the Col and NS groups were $2.5(0-5)$ and $0.5(0.5-3)$, respectively $(\mathrm{p}=0.06)$.

\section{Bacterial resistance rates}

During the study period there were no differences in the presence of colistin-resistant bacteria between groups; there was, however, a significant decrease in GNB-TBA isolates (table 3). The frequency of colistin-sensitive isolates, in particular those with lowest minimum inhibitory concentration value for colistin $\left(\leqslant 0.5 \mu \mathrm{g} \cdot \mathrm{mL}^{-1}\right)$, was found to be increased in the post-intervention period compared with the pre-intervention period (online supplementary table S6).

\section{Side effects}

Two patients developed bronchospasm attributed by physicians to normal saline and eight patients developed bronchospasm after colistin inhalation $(\mathrm{p}=0.10)$; in all but one case, bronchospasm was mild and managed with inhaled $\beta$-agonists; in one case, the attending physician decided on the termination of colistin nebulisation on day 6. 


\begin{tabular}{|c|c|c|c|c|}
\hline & Overall & Col group & NS group & p-value \\
\hline Overall isolates & $194(100)$ & $97(100)$ & 97 (100) & \\
\hline Total TBA isolates & $130(67)$ & 60 (61.9) & $70(72.2)$ & 0.17 \\
\hline Total blood isolates & $64(33)$ & $37(38.1)$ & $27(27.8)$ & 0.17 \\
\hline MDR TBA isolates & $79(40.7)$ & $30(30.9)$ & 49 (50.5) & 0.01 \\
\hline Airway colonisation cases & $48(24.7)$ & $20(20.6)$ & $28(28.9)$ & 0.24 \\
\hline VAT or VAP cases & $31(16)$ & 10 (10.3) & $21(21.6)$ & 0.05 \\
\hline Gram-positive TBA isolates & $7(3.6)$ & $3(3.1)$ & $4(4.1)$ & 1.0 \\
\hline Gram-negative TBA isolates & $72(37.1)$ & $27(27.8)$ & $45(46.4)$ & 0.01 \\
\hline MDR blood isolates & 49 (25.2) & $26(26.8)$ & $23(23.7)$ & 0.74 \\
\hline Gram-positive isolates & $8(4.1)$ & $6(6.2)$ & $2(2.1)$ & 0.28 \\
\hline Gram-negative isolates & $41(21.1)$ & $20(20.6)$ & $21(21.6)$ & 1.0 \\
\hline Colistin-resistant TBA isolates & $44(22.7)$ & $25(25.8)$ & $19(19.6)$ & 0.39 \\
\hline Airway colonisation cases & $10(5.1)$ & $7(7.2)$ & $3(3.1)$ & 0.33 \\
\hline VAT or VAP cases & $14(7.2)$ & $6(6.2)$ & $8(8.2)$ & 0.78 \\
\hline Gram-positive TBA isolates & $10(5.1)$ & $6(6.2)$ & $4(4.1)$ & 0.75 \\
\hline Gram-negative TBA isolates & $14(7.2)$ & $7(7.2)$ & 7 (7.2) & 1.0 \\
\hline Colistin-resistant blood isolates & 20 (10.3) & $12(12.4)$ & $8(8.2)$ & 0.48 \\
\hline Gram-positive isolates & $8(4.1)$ & $6(6.2)$ & $2(2.1)$ & 0.28 \\
\hline Gram-negative isolates & $12(6.27)$ & $6(6.2)$ & $6(6.2)$ & 1.0 \\
\hline
\end{tabular}

All data are presented as $\mathrm{n}(\%)$. MDR: multidrug-resistant; TBA: tracheobronchial aspirate; Col group: colistin group; NS group: normal saline group; VAT: ventilator-associated tracheobronchitis; VAP: ventilator-associated pneumonia. \#: polymicrobial cases containing Gram-negative microbes were included in the Gram-negative group.

\section{Discussion}

In the present randomised study, the 30-day incidence of VAP was not significantly different between patients who received nebulised colistin for their first $10 \mathrm{ICU}$ days as prophylaxis for VAP and patients who received nebulised normal saline for the same period.

Few studies have investigated pneumonia prophylaxis with antibiotics administered to the airways in critical care [9-11]. Rouby et al. [9] instilled colistin within the tracheal tube and decreased the incidence of bronchopneumonia due to GNB; however, they used digestive selective decontamination in addition to tracheal instillation. KLICK et al. [10] sprayed polymyxin B in the trachea/pharynx and found decreased GNB-pneumonia in a mixed population of intubated and non-intubated patients. In the present study, we used nebulised colistin in mechanically ventilated patients that might improve antibiotic delivery in the lungs and we did not use digestive decontamination in order to eliminate the effects of that strategy [21] on the outcomes assessed. However, the effect of the prophylaxis on VAP incidence was not significantly different between groups.

We used a daily dose of $1.5 \times 10^{6} \mathrm{U}$ colistimethate sodium ( $45.5 \mathrm{mg}$ colistin base activity) for 10 days or until extubation. The dose was similar to the prophylactic dose used previously $\left(1.6 \times 10^{6} \mathrm{U}\right.$ daily, by endotracheal installation, for 15 days) [9]. These dosing regimens are approximately half the usual daily treatment dose of VAP by nebulisation $\left(2-6 \times 10^{6} \mathrm{U}\right)[22,23]$. Currently, there is no known optimum dose, duration or modality of administration of colistin for the prophylaxis [9-11] or the treatment of VAP [24, 25]. Data are sparse [23-25] and the evaluation of the pharmacokinetics of colistin is complex [26-29]. A future investigation may further refine the optimum dosing and duration of prophylaxis/treatment of VAP.

One might also argue that inhaled saline may have an impact on VAP rates in the NS group. Saline might decrease both tube biofilm formation and secretion clearance, which could prevent pneumonia [30]. However, the administration of nebulised saline requires a temporal disconnection of the ventilator circuit (as is also the case for the Col group), and might favour the contamination of the airways by microorganisms and likely increase VAP rates in the control group. Nevertheless, VAP frequency in the NS group was decreased compared with VAP frequency found in the control group in our previous study [13].

Another potential explanation for the negative results regarding the primary outcome in this study could be the lack of power to detect such an effect; the VAP rate in the control group of this study was lower than expected based on the data of our previous study used for power calculation [13]. A different primary end-point, such as GNB-VAP incidence, might have been more appropriate. In fact, the GNB-VAP rate, 
which was assessed secondarily, was significantly lower in the Col group compared with the NS group. Interestingly, other secondary outcomes, such as VAP incidence due to A. baumannii or VAP IDR, were lower in the Col group (table 2). Cox regression analysis also suggested that inhaled colistin prophylaxis was an independent factor for decreased VAP IDR, GNB-VAP and MDR-VAP.

VAT and colonisation rates were similar in the Col and NS groups over the 30-day period assessed. However, the Col group presented lower colonisation rates during the intervention period, but higher rates following prophylaxis, suggesting a rebound phenomenon that merits further investigation in the future. A recent study [31] found aerosolized antimicrobials to be associated with a reduction of MDR bacteria in the respiratory tract. However, there were methodological differences (treatment method and antibiotics), and detailed data on respiratory cultures beyond the 15-day treatment period were not available and a direct comparison might be difficult.

FEELEY et al. [11] reported the emergence of polymyxin-resistant organisms and also an excessive pneumonia mortality of $64 \%$ after the administration of polymyxin B to intubated and spontaneous breathing patients (intratracheally and as aerosol in the upper airways) during their entire ICU stay. In our study, we administered colistin by nebulisation only in intubated patients for a 10-day period and prophylaxis was not associated with ICU mortality. Nevertheless, we noticed that among patients who presented VAP, ICU mortality was decreased. A plausible explanation for such an effect on this population might be attenuation in airway inflammation during the course of VAP. In agreement with this, the SOFA score on the day of infection diagnosis and the white blood cell count following VAP diagnosis were found to be significantly decreased in the Col group compared with the NS group.

In the present study, many patients were on systemic antibiotics covering GNB during the period of prophylaxis with colistin. According to our findings, patients who received systemic antibiotics covering GNB plus inhaled colistin presented significantly less VAP or GNB-VAP compared with patients who received similar systemic antibiotics plus inhaled normal saline. In this respect, the use of inhaled colistin might have an impact on VAP even in the presence of systemic antibiotics covering GNB.

We proceeded in a post hoc analysis of VAP rates in neurosurgical patients, which represented a large percentage of our population; in our previous study, neurosurgical admission was an independent risk factor for VAT [6]. In this study, neurosurgical admission was independently associated with GNB-VAP, whereas GNB-VAP was lower in neurosurgical Col group patients (see online supplementary material). On this basis, neurocritical patients might represent a target group for prophylactic administration of inhaled colistin.

\section{Study limitations}

The present study has also some other points that should be taken into consideration when interpreting the results. First, VAP diagnosis was based on previously accepted definitions and bacterial thresholds in bronchial secretions $[17,18]$. However, in the Col group, the levels of bacterial growth might be influenced by high concentrations of antibiotics in the airway. In order to clarify this point, we additionally analysed our data by using only clinical criteria for VAP, regardless of the presence of bacteria in TBA (see online supplementary material); both VAP incidence and VAP IDR were lower in the Col group compared with the NS group. Second, we evaluated the ecological impact of our intervention by assessing the incidence of resistance against colistin in bronchial and blood samples in certain periods of the study; weekly surveillance cultures (including stool and pharyngeal samples) were not performed during the entire study period due to a limitation of resources. Thus, we acknowledge that the study may lack power to detect such an effect. Third, this is an open-label, single-centre study, in an ICU with predominantly GNB flora; the department serves many neurosurgical patients who might require a prolonged stay and the number of screened patients may be relatively small for the given period. Furthermore, patients with severe COPD or pneumonia on admission were excluded from the study as they may have excessive secretions obscuring respiratory infection diagnosis or the assessment of resolution of the infection.

In conclusion, the present investigation suggests that the prophylactic administration of nebulised colistin in critical care patients did not reduce overall VAP incidence.

\section{Acknowledgements}

The authors thank Mrs Christina Sotiropoulou (Statistician; Thorax Institute, Athens, Greece) for her assistance in statistical analyses and Ross Robertson (BSc Microbiology; Garagani-Robertson English Schools, Volos, Greece) for his proofreading of the original manuscript.

\section{References}

Chastre J, Fagon JY. Ventilator-associated pneumonia. Am J Respir Crit Care Med 2002; 165: 867-903.

Rello J, Ollendorf DA, Oster G, et al. VAP Outcomes Scientific Advisory Group: epidemiology and outcomes of ventilator-associated pneumonia in a large US database. Chest 2002; 122: 2115-2121. 
3 Bercault N, Boulain T. Mortality rate attributable to ventilator-associated nosocomial pneumonia in an adult intensive care unit: a prospective case-control study. Crit Care Med 2001; 29: 2303-2309.

4 ATS. Guidelines for the management of adults with hospital-acquired, ventilator-associated, and healthcare-associated pneumonia. Am J Respir Crit Care Med 2005; 171: 388-416.

5 Bouderka MA, Fakhir B, Bouaggad A, et al. Early tracheostomy versus prolonged endotracheal intubation in severe head injury. J Trauma 2004; 57: 251-254.

6 Karvouniaris M, Makris D, Manoulakas E, et al. Ventilator-associated tracheobronchitis increases the length of intensive care unit stay. Infect Control Hosp Epidemiol 2013; 34: 800-808.

7 Nseir S, Blazejewski C, Lubret R, et al. Risk of acquiring multidrug resistant Gram-negative bacilli from prior room occupants in the intensive care unit. Clin Microbiol Infect 2011; 17: 1201-1208.

8 Palmer LB, Smaldone GC, Chen JJ, et al. Aerosolized antibiotics and ventilator-associated tracheobronchitis in the intensive care unit. Crit Care Med 2008; 36: 2008-2013.

9 Rouby JJ, Poete P, Martin de Lassale E, et al. Prevention of Gram-negative nosocomial bronchopneumonia by intratracheal colistin in critically ill patients. Histologic and bacteriologic study. Intensive Care Med 1994; 20: 187-192.

10 Klick JM, du Moulin GC, Hedley-Whyte J, et al. Prevention of gram-negative bacillary pneumonia using polymyxin aerosol as prophylaxis. II. Effect on the incidence of pneumonia in seriously ill patients. J Clin Invest 1975; 55: 514-519.

11 Feeley TW, Du Moulin GC, Hedley-Whyte J, et al. Aerosol polymyxin and pneumonia in seriously ill patients. N Engl J Med 1975; 293: 471-475.

12 Whonet Greece. Cumulative results of Jan-Jun 2012. http://www.mednet.gr/whonet. Date last accessed: June 26, 2013.

13 Makris D, Manoulakas E, Komnos A, et al. Effect of pravastatin on the frequency of ventilator-associated pneumonia and on intensive care unit mortality: open-label, randomized study. Crit Care Med 2011; 39: 2440-2446.

14 Global Initiative for Chronic Obstructive Lung Disease (GOLD). Global strategy for diagnosis, management, and prevention of COPD. http://www.who.int/respiratory/copd/GOLD_WR_06.pdf. Date last accessed: September 21, 2011.

15 Ari A, Areabi H, Fink JB. Evaluation of aerosol generator devices at 3 locations in humidified and non-humidified circuits during adult mechanical ventilation. Respir Care 2010; 55: 837-844.

16 Mead R, Curnow R. Statistical methods in agriculture and experimental biology (Texts in statistical science). New York, Chapman \& Hall, 1983; p. 34.

17 Craven DE, Chroneou A, Zias N, et al. Ventilator-associated tracheobronchitis (VAT): the impact of targeted antibiotic therapy on patient outcomes. Chest 2008; 135: 521-528.

18 Nseir S, Favory R, Jozefowicz E, et al. Antimicrobial treatment for ventilator-associated tracheobronchitis: a randomized controlled multicenter study. Crit Care 2008; 12: R62.

19 Bouadma L, Deslandes E, Lolom I, et al. Long-term impact of a multifaceted prevention program on ventilator-associated pneumonia in a medical intensive care unit. Clin Infect Dis 2010; 51: 1115-1122.

20 Makris D, Moschandreas J, Damianaki A, et al. Exacerbations and lung function decline in COPD: new insights in current and ex-smokers. Respir Med 2007; 101: 1305-1312.

21 de Smet AM, Kluytmans JA, Cooper BS, et al. Decontamination of the digestive tract and oropharynx in ICU patients. N Engl J Med 2009; 360: 20-31.

22 Michalopoulos A, Papadakis E. Inhaled anti-infective agents: emphasis on colistin. Infection 2010; 38: 81-88.

23 Athanassa ZE, Markantonis SL, Fousteri M-Z, et al. Pharmacokinetics of inhaled colistimethate sodium (CMS) in mechanically ventilated critically ill patients. Intensive Care Med 2012; 38: 1779-1786.

24 Rattanaumpawan P, Lorsutthitham J, Ungprasert P, et al. Randomized controlled trial of nebulized colistimethate sodium as adjunctive therapy of ventilator-associated pneumonia caused by Gram-negative bacteria. J Antimicrob Chemother 2010; 65: 2645-2649.

$25 \mathrm{Lu}$ Q, Luo R, Yang J, et al. Efficacy of high-dose nebulized colistine in ventilator-associated pneumonia caused by multidrug resistant Pseudomonas aeruginosa and Acinetobacter baumannii. Anesthesiology 2012; 117: 135-147.

26 Rouby JJ, Bouhemad B, Monsel A, et al. Aerosolized antibiotics for ventilator-associated pneumonia. lessons from experimental studies. Anesthesiology 2012; 117: 1364-1380.

27 Kassamali Z, Rotschafer JC, Jones RN, et al. Polymyxins: wisdom doesn't always come with age. Clin Infect Dis 2013; 57: 877-883.

28 Ratjen F, Rietschel E, Kasel D, et al. Pharmacokinetics of inhaled colistin in patients with cystic fibrosis. J Antimicrob Chemother 2006; 57: 306-311.

29 Lu Q, Girardi C, Zhang M, et al. Nebulized and intravenous colistin in experimental pneumonia caused by Pseudomonas aeruginosa. Intensive Care Med 2010; 36: 1147-1155.

30 Caruso P, Denari S, Ruiz S, et al. Saline instillation before tracheal suctioning decreases the incidence of ventilator-associated pneumonia. Crit Care Med 2009; 37: 32-38.

31 Palmer LB, Smaldone GC. Reduction of bacterial resistance with inhaled antibiotics in the intensive care unit. Am J Respir Crit Care Med 2014; 189: 1225-1233. 